Pathologe $2010 \cdot 31: 329-330$

DOI 10.1007/s00292-010-1374-2

(c) Springer-Verlag 2010

\author{
K.-M. Müller \\ Gerhard-Domagk-Institut für Pathologie, Universitätsklinikum Münster
}

\title{
Zur Übergabe der Schriftleitung
}

Die letzten 10 Jahre waren im Redak-

Seit der Gründung von Der Pathologe durch Herrn Prof. Dr. Volker Becker (Erlangen) im Jahre 1979 sind mehr als 30 Jahre vergangen. In einem Editorial in Der Pathologe 3/2009 hat Frau Althaus einen Rückblick auf diese Zeit gegeben [1].

Vor 16 Jahren (1994) wurde - unter anderem auf Initiative von Herrn Dr. Thiekötter vom Springer-Verlag - in Frankfurt eine neue Struktur für die Schriftleitung von Der Pathologe beschlossen. Dem federführenden Schriftleiter, seinerzeit Prof. Dr. W. Böcker (Münster), wurde eine Gruppe von 10 Fachexperten zu speziellen Themen und Fragestellungen der Pathologie an die Seite gestellt. Das zunächst als Experiment geplante Projekt hat sich seit 1994 bewährt und wird begründet fortgeführt. Das Expertengremium der erweiterten Schriftleitung bildete eine wesentliche Grundlage für die seit Jahren etablierte Erstellung von überwiegend organbezogenen Schwerpunktheften und aktuellen Themenheften von Der Pathologe termingerecht zu den jährlichen Symposien der Internationalen Akademie für $\mathrm{Pa}$ thologie (IAP) in Bonn.

Diese positive Entwicklung war nur durch den unermüdlichen Einsatz aller im Schriftleitergremium gemeinsam arbeitenden Kolleginnen und Kollegen möglich. Ihnen und zahlreichen zusätzlichen Gutachtern gilt für die gute und erfolgreiche Zusammenarbeit großer Dank und Anerkennung. Den Damen des Verlages Frau Dr. Koch und Frau Althaus - sei für die immer sehr gut vorbereiteten Herausgebersitzungen im Februar in Bonn gedankt. In stets harmonischer Atmosphäre erfolgten intensive Diskussionen und Planungen. Hier wurden in den vergangenen 11 Jahren auch freundschaftliche Kontakte als vertrauensvolle Basis für die gemeinsamen Anliegen geschaffen. tionsbereich auch geprägt vom Einzug der elektronischen Medien zur Aufbereitung der Manuskripte und zahlreicher Abbildungen. Kontinuierlich mussten Übergänge von der „Papierform“ mit „Hochglanzabbildung“ über Arbeiten auf Datenträgern mit Disketten, CDs und USBSticks bis zur jüngsten „Online-Einreichung“ von Beiträgen vollzogen werden. In diesem Zusammenhang sei die jeweils hervorragende kostenneutrale Druckqualität der Abbildungen zu den Arbeiten hervorgehoben. Und bei der kontinuierlichen Neu- und Umgestaltung der Titelseite in den letzten Jahren hat der Verlag ebenfalls viel Eigeninitiative eingebracht.

Die Schriftleitung konnte und kann jederzeit auf die sehr gute Unterstützung und Beratung des Verlages zurückgreifen, wobei die jahrelangen stets harmonischen und auch in schwierigen Situationen stimulierenden und fördernden Hilfestellungen von Frau Althaus und Frau Dr. Koch sowie ihrer Vorgängerinnen wesentliche Grundlage für den erfolgreichen Abschluss von 70 Heften waren.
Abb. 1 Nach 11 Jahren übergibt Herr Prof. Dr. K.-M. Müller die federführende Schriftleitung an Herrn Prof. Dr. K.W. Schmid.

(Foto mit freundlicher Genehmigung von Robin A. Cooke)
Wiederholt wurden in den letzten Jahren - auch vor dem Hintergrund elektronisch verfügbarer Fachbibliotheken Fragen der Daseinsberechtigung und $\mathrm{Zu}$ kunft einer deutschsprachigen Fachzeitschrift für Pathologie in gedruckter Form gestellt. Die aktuelle Antwort dazu haben Abonnenten und Leser bei einer Umfrage des Verlages anlässlich des IAP-Symposiums in Bonn 2009 gegeben. Mit großer Mehrheit wurde die interessante und praxisnahe Darstellung von gesichertem Wissen in Der Pathologe hervorgehoben. Als „sehr wichtig“ bzw. „wichtig“ bewertet wurden die Hefte mit Themenschwerpunkten (seit 2001); auch Übersichten, Originalarbeiten und Kasuistiken genießen einen hohen Stellenwert. Großes Interesse besteht außerdem an kommentierter Originalliteratur, Diskussion neuer Methoden, Arbeiten über Aufbereitung und Erstellung von täglichen Begutachtungen sowie Leitlinien. Es ist zu erwarten, dass sich die allmähliche Etablierung der neuen Medien in Format und Präsentation der Inhalte von Der Pathologe in diesem Sinne positiv niederschlägt.

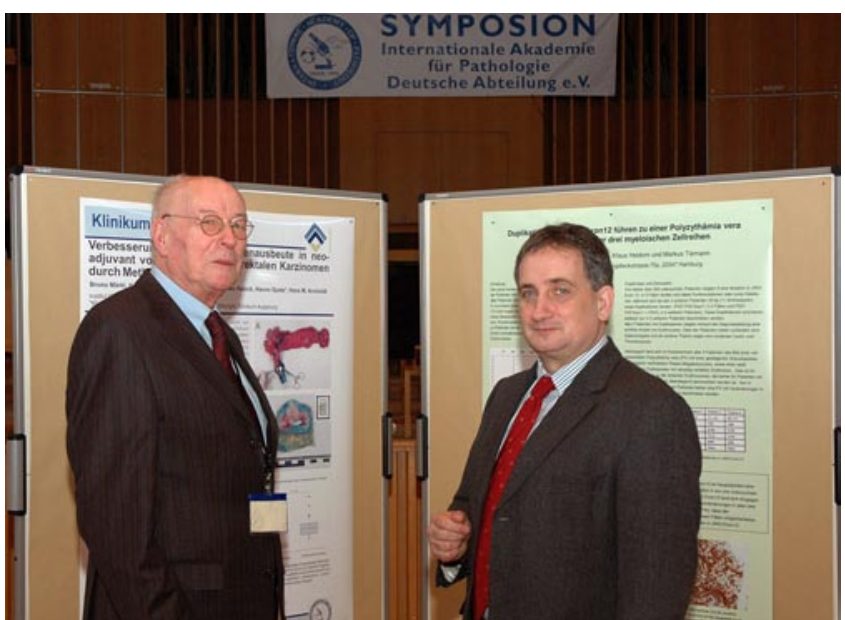


Grundsätzlich stellt der Verlag jederzeit Leitfäden für Autoren zur Verfügung; diese sind zu finden unter http:// www.derpathologe.de. Hier können Manuskripte über das elektronische System „Editorial Manager“ eingereicht werden. Alle Autoren sind eingeladen, Der Pathologe mit ihren persönlichen Arbeiten zu bereichern!

Eine wesentliche Erweiterung des wissenschaftlichen Spektrums von $\mathrm{Der} \mathrm{Pa}$ thologe wurde seit dem Jahre 2008 durch Sonderausgaben im Rahmen der Jahrestagungen der Deutschen Gesellschaft für Pathologie (DGP) erzielt. Sie führen die bisher in der Reihe der „Verhandlungsbände“ der DGP gedruckten Zusammenfassungen der Ansprachen, der wissenschaftlichen und fachspezifischen Ergebnisse, der Hauptreferate und Berichte aus den Arbeitsgemeinschaften sowie der Sitzungsprotokolle und Nachrufe der DGPJahrestagungen kontinuierlich fort.

Nach 11 Jahren geht die federführende Schriftleitung von Bochum nach Essen nun in neue Hände über ( $\bullet$ Abb. 1). Mein großer Dank gilt allen Mitarbeitern des Bochumer Institutes. Dabei muss der unermüdliche, diplomatische und erfolgreiche Einsatz der Sekretariatsleiterin Frau Elke Griffiths und des Assistenten der Schriftleitung Prof. Dr. C. Kuhnen für Der Pathologe besonders hervorgehoben und gewürdigt werden. Allen vielen herzlichen Dank!
Dem neuen Schriftleiter, Herrn Prof. Dr. Kurt Werner Schmid, und seiner Mannschaft im Editorial Board gelten unsere guten Wünsche und ein herzliches "Glück auf " für eine gute, erfolgreiche und schöne Zukunft von Der Pathologe.

Im September 2010

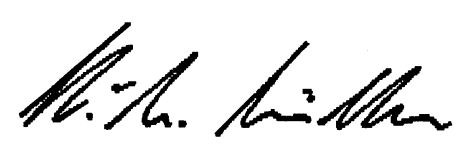

Prof. Dr. K.-M. Müller

\section{Korrespondenzadresse}

Prof. Dr. K.-M. Müller

Gerhard-Domagk-Institut für Pathologie,

Universitätsklinikum Münster

Domagkstr. 17, 48149 Münster

klausmichael.mueller@ukmuenster.de

\section{Literatur}

1. Althaus E (2009) 30 Jahre Der Pathologe. Pathologe 30:171-172

\section{Neue „Starting Grants“ des European Research Council}

Der European Research Council (ERC) hat nun einen neuen Ausruf für die ERC „Starting Grants" gestartet. Dieses Förderinstrument wendet sich an herausragende Forscherpersönlichkeiten, die eine Forschungsgruppe aufbauen bzw. konsolidieren möchten.

Wissenschafterlnnen aus allen Disziplinen und aller Nationalitäten können sich für den Starting Grant bewerben. Voraussetzung ist jedoch, dass die Forschungstätigkeiten in einem der EU Mitgliedsstaaten oder in einem assoziierten Land durchgeführt werden. Die Ausschreibung sieht kein Alterslimit vor; AntragsstellerInnen müssen jedoch mindestens 2 und maximal 12 Jahre vor der Veröffentlichung der Ausschreibung ihr Doktorat abgeschlossen haben. Starting Grants werden mit Summen von bis zu 1.500.000 EUR für eine Dauer von maximal 5 Jahren gefördert (unter bestimmten Voraussetzungen sind bis zu 2 Mio EUR beantragbar). Der ERC zielt auf die Förderung von so genannter "frontier research", Forschung, durch die die Grenzen des Wissens, unabhängig von disziplinären Grenzen, erweitert werden.

Die Anträge können über das „Electronic Proposal Submission System“ (EPSS) eingereicht werden. Die Deadline für Anträge ist abhängig vom Wissenschaftsgebiet:

- Physical Sciences \& Engineering:

14. Oktober 2010 (17 Uhr)

- Life Sciences: 9. November 2010 (17 Uhr)

- Social Sciences \& Humanities:

24. November 2010 (17 Uhr)

Quelle: FWF-Der Wissenschaftsfonds, www.fwf.ac.at

Zum Abschied

Der Verlag möchte an dieser Stelle dem langjährigen Schriftleiter für seine hervorragende Arbeit für Der Pathologe danken. Herr Professor Müller hat die Zeitschrift mit großem Engagement geführt und durch seine fachliche und persönliche Kompetenz sehr geprägt. Wir freuen uns, Der Pathologe ein Stück weit gemeinsam begleitet und gestaltet zu haben, und wünschen Herrn Professor Müller für die Zukunft alles Gute!

Für die Redaktion

E. Althaus

B. Koch 